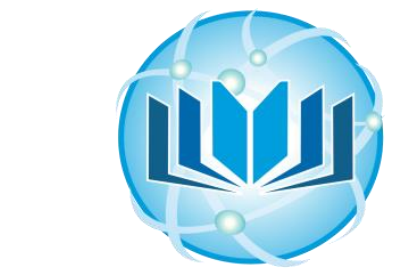

\title{
COVID-19 and Dementia: A Review and Synthesis of Material on a Deadly Combination Nasreen Basoudan ${ }^{1 *}$ and Andrea Tales ${ }^{2}$
}

\section{Affiliation}

${ }^{1}$ Department of Psychology, Swansea University, Swansea, UK

${ }^{2}$ Centre for Innovative Ageing, Swansea University, Swansea, UK

*Corresponding author: Nasreen Basoudan, Department of Psychology, Swansea University, Swansea SA2 8PP, UK, Tel: +966506636618, E-mail: nasreen.s.basoudan@ gmail.com

Citation: Basoudan N and Tales A. COVID-19 and dementia: a review and synthesis of material on a deadly combination (2020) Neurophysio and Rehab 3: 11-15.

Received: Sep 23, 2020

Accepted: Oct 26, 2020

Published: Nov 02, 2020

Copyright: () 2020 Basoudan N, et al., This is an open-access article distributed under the terms of the Creative Commons Attribution License, which permits unrestricted use, distribution, and reproduction in any medium, provided the original author and source are credited.

\section{Abstract}

Within modern history, the majority of the worldwide population has not experienced pandemic conditions, and not in recent history has a global pandemic occurred. Arguably therefore we were not prepared for, and did not anticipate, some of its effects, with emerging evidence indicating that this was particularly the case with respect to dementia care. The aim of this paper is to highlight some of the issues raised by COVID-19 surrounding dementia care, focusing on issues experienced both by individuals living with dementia and by those who provide their care. This is in response to the need to raise awareness of this issue and we do so by bringing this information together in order that it might inform policy for ongoing care post-virus and future outbreaks and lockdown situations. While there is a marked need to change the current tactics being used in the treatment and care of preexisting and longstanding illnesses, like dementia, there is also a need to take into account the safety procedures necessary to prevent potential exposure to COVID-19. Through the targeted exploration of these considerations, there is a hope that documentation of these actions can both facilitate current treatment and care efforts and approaches and provide a framework for future efforts, should another pandemic situation arise in the near future.

Keywords: Dementia, COVID-19, Treatment, Ongoing care, Procedures

Abbreviations: CNS-Central Nervous System, PNS-Peripheral Nervous System.

\section{Introduction}

COVID-19, a novel coronavirus, also known as SARS-CoV-2, has spread around the globe, resulting in a large number of infected people, a high mortality rate, and leaving those who do recover suffering from a host of complications brought about by the viral infection. The effects of the virus upon the respiratory systems of patients has been labelled a primary concern, though significant other concerns are starting to manifest including clotting disorders, strokes, and neurological involvement. Neurological involvement can present with both Central Nervous System (CNS) and Peripheral Nervous System (PNS) effects, resulting in some cases in demyelination and symptomology in alignment with dementia. Although much still remains unknown about the health-related effects of COVID-19, it is clear that the pandemic has dramatically affected how most people act, react, respond, and interact with society in general, with evidence emerging to suggest that these effects are particularly detrimental for older adults and especially those living with dementia and carers. Below, we discuss some of the potential reasons for these additional difficulties [1-7].

Governmental response to the pandemic has varied from country to country, yet for the majority of European countries, governmental efforts intended to control the spread of the virus have been similar. Some countries have implemented practices intended to combat the spread of the virus within the elderly population.
These efforts include the decision to close long-term care facilities, including those responsible for the provision of professional care to patients with dementia, and to all non-essential persons, meaning that family members and friends are unable to visit the individual housed within the care facility. While this response to COVID-19 was understandable in the face of evidence available during the early stages of the pandemic, there is now a marked need to explore the effects of COVID-19 on those already being served by the healthcare community prior to the pandemic, in light of emerging evidence indicating the potential detrimental effects of this policy upon dementia care, in order that any resurgence and associated policy can take these effects into consideration. The purpose of this literature review therefore is to explore the relationship between COVID-19 and dementia, focusing on both the provision of care to patients with dementia during the pandemic and the effects of the pandemic on carers [8].

Shea and DeKosky offered an explanation of the ways in which services have broken down for patients with dementia as a result of the COVID-19 pandemic, highlighting the ways in which efforts were being made to facilitate continuation of care, including the use of volunteers, due to a lack of available staffing and a need to quarantine staff who have, in spite of the implementation of best practices, been exposed to the virus. 
The researchers note that the solutions that have been implemented are not perfect, or even adequate, considering that while volunteers are useful, there is no guarantee that they are following best practices in prevention of exposure. Still further, there is no current prognosis for COVID-19 in patients with dementia, or patients with any form of cognitive impairment, creating the potential for greater risk within this population [9].

\section{Dementia}

Dementia is a term that is used to describe a grouping of symptoms that have the potential to affect the cognitive processes and social capabilities of the individual to the point where daily functionality becomes compromised. Carers therefore play an important part of the life of someone living with dementia. In some instances, carers are family members, while in others, where round the clock care may be needed, professional facilities and the services of professional carers are utilized in order to facilitate the health, care and quality of life for those living with dementia. This arrangement, of family members providing care where they are able, and professional services stepping in to offer care where they are not, is one that has been in existence for decades, yet arrangements of this nature have not previously been interrupted by a global pandemic as they are currently [1,2,10-14].

Although the effects of COVID-19 on care homes has been the subject of much research and a great deal of media attention, only limited information is available, and limited research has been conducted, on the effects of COVID-19 on patients with dementia and carers living at home under lockdown conditions [15-18].

\section{Dementia Care during the COVID-19 Pandemic}

Martin-Jimenez et al., [19] examined the cognitive impairments identified in those who later died with COVID-19 and noted that there was an increased frequency of patients who died of COVID-19 within a hospital setting who had a preexisting diagnosis of either dementia or mild cognitive impairment, as compared to those without diagnosis. There is still little that is known regarding the true extent of cognitive impairment and its relationship to mortality rates associated with the disease; to this end the researchers sought to compare the comorbidities of patients who died of COVID-19. Martin-Jimenez et al., noted that of the 477 cases of patients who died of COVID-19 between the first of March and the 31st of March of 2020,30\% of patients displayed cognitive impairment, another $21.1 \%$ had a diagnosis of dementia, and $8.9 \%$ of the sample showed mild cognitive impairment, suggesting that demyelination had not advanced sufficiently to a diagnosis of cognitive impairment or dementia before the patient succumbed [19].

With COVID-19's neurological involvement, patients already diagnosed with dementia prior to the pandemic appear particularly susceptible to COVID-19 due to a combination of factors, including neurological complications, manifestations of neurological symptoms associated with viral infection, and co-morbidities associated with preexisting neurological symptoms. This presentation has resulted in unique challenges to neurologists including increased effects of demyelination, difficulties identifying which symptoms may be manifesting as a result of comorbidities, which symptoms are manifesting as a result of viral infection, and which symptoms are increasing in severity as a result of disease progress, among other considerations. As dementia is one of the most common comorbidities in deceased patients it has been suggested that patients already diagnosed with dementia may be less likely to receive care given their quality of life conditions and the lack of available and necessary medical supplies needed to treat the associated symptomology of COVID-19; with additional concerns that preferential treatment would be given to COVID-19 patients who were younger and who displayed less severe comorbidities than those living with dementia due to their potential utility in society $[3,20,21]$.

Bhaskar et al., [22] expressed a similar concern with respect to the neurological components of COVID-19, arguing that while neurologists have received the benefit of knowledge obtained from caring for patients with dementia diagnosed with COVID-19, thereby facilitating societal understanding of disease progress, they had noted a reorganization of services and a triaging of provided care to patients with COVID-19 based on decreases in available resources and an overburdening of healthcare systems. However, this information pertains to those in care homes, as researchers currently lack an effective method of collecting data from patients who reside with their carers in at-home conditions during the lockdown. To this end, there is a need to gather this evidence in a unified body of knowledge. A continually growing body of evidence shows neurological involvement as a symptom of COVID-19 [3,19, 21,22].

In light of these findings, there is a need to explore the relationship between dementia and COVID-19. While evidence of this nature is sparse, researchers have an obligation to explore these concerns, particularly when there is a positive correlation between COVID-19 mortality and dementia, suggesting that those with a preexisting condition in which cognitive impairment is present may experience an increased risk of contracting COVID-19. Dementia and COVID-19 issues have tended to be focused on medical/health and neurological considerations, but there is also a need to focus on the evidence concerning care for people living with dementia, particularly as it pertains to at home care and care in a care setting [23].

As a global society, "we are witnessing the huge effects of the present corona (virus) pandemic". While there are those who hypothesize that the virus will disappear soon, in spite of the lack of evidence supporting such a supposition, even these individuals indicate that there is a need to address care for patients with dementia in spite of the pandemic. This is particularly the case with respect to further outbreaks or a need for additional lockdowns. Korczyn highlighted the need to provide physical contact to those living with dementia, and to maintain visiting and memory exercises to as high a degree possible in order to prevent further deterioration, both for those in care facilities and those in care home settings [24]. Korczyn also reminded readers that there is a need to explore the more serious concerns of whether hospitalization in the event of injury associated with people living with dementia, based on the potential risk for these patients to contract the virus. Behavioral changes to routine are recommended, along with the integration of additional preventative care options [24,25].

However, while the underlying premise of attempting to reduce risk to patients with dementia and attempting to reduce possible COVID-19 exposure in these patients through behavioral modification and increased preventative care sounds good on paper, the ability to implement these suggestions within an active environment is far more difficult than it may initially appear. This serves to highlight another area in which there simply is not enough information available as of yet; the focus has been placed on understanding the disease, as opposed to concentrating on coping strategies and the implementations thereof.

Telehealth practices, including the provision of additional care and supervision through virtual communication are not uncommon, and with the COVID-19 pandemic, many facilities have turned to the use of these tools as a means of decreasing the risk of person-to-person contact. In particular, the use of telehealth has increased in the provision of care to those in nursing homes and other facilities providing care to older patients and those diagnosed with dementia. However, while some benefit is afforded, including the continuation of care, albeit in a different form from what was previously utilized; there are marked differences in the provision of care that may be difficult for patients used to routine to acclimate [26]. 
This decreased person-to-person contact has resulted in behavioral changes within patients with dementia who receive care in both care homes and within the at home environment, resulting in increased anxiety, irritability, and manifestations of difficult behaviors due to the change in routine brought about by this shift. Depending on the resources of the facility, some patients with dementia are still able to communicate virtually with family members, though even those who are receiving telehealth services and virtual communication with family members and friends are displaying increased symptoms of anxiety, irritability, depression, and apathy, along with difficulties sleeping and loss of appetite, among other adverse considerations. While this loss of direct person-to-person contact may be reducing risks for certain issues, it has increased the risk of others. This information, however, provides key insights into the non-physical impacts of the disease, impacts that extend beyond the treatment of the virus and serves to highlight an area in which additional preparation is needed [27].

Wang et al., [28] pointed out that patients with dementia do already experience cognitive difficulties, which may seem to be a redundant note, but this extends far beyond not remembering what day of the week it is. People living with dementia may not remember that there even is a pandemic, causing additional confusion beyond the loss of person-to-person contact and extending to the inability to utilize basic safeguarding procedures, being unable to remember to wear a mask, or even being unable to remember to wash their hands frequently. For those who do not live in professional care facilities, this not only creates an additional risk to the dementia patients themselves, it creates additional risk to the carers responsible for the health and safety of these patients. Loss of understanding of how to utilize basic technology can further compound and complicate this problem, as not only is the day to day routine for these individuals disrupted completely, they are also faced with the difficult challenge of utilizing unfamiliar technologies. An inability to use telehealth or virtual communication technologies, compounded with cognitive impairment means that even if these individuals are able to utilize the technologies on one day, they may not remember how to do so on subsequent days, creating still further challenges. Of further consideration is that while telehealth technologies may be readily available, or at least more readily available, in care homes, accessibility to telehealth by carers within the home setting will be limited based on the technologies available within the home, further compounding the situation. A tool, no matter how beneficial, is ineffective if it is inaccessible to those who need it [28].

It is not only a change in the type of care provided, or a lack of visitors, which, in turn, can have an adverse effect on the memories of those diagnosed with dementia; all aspects associated with the provision of care to patients with dementia have changed because of the pandemic. Older adults are already experiencing difficulties receiving access to oral healthcare, a necessary and preventative health service, and this matter is compounded for patients with dementia due to the additional amount of carers needed, and additional staff needed, in order to facilitate the receipt of care. Socioeconomic problems within the population living with dementia were already compounded by lack of access to care, reduced autonomy, and additional comorbidities. This combined with a loss of activities of daily living and a generally declining ability to maintain oral health standards, means that access to oral healthcare is of particular importance to this population. Yet, because of the additional difficulties associated with transport and assistance of dementia patients in order to even get to the oral healthcare office, this poses an additional problem in a time of social distancing, declined social interaction, and declined social contact, particularly when faced with saliva, a means of viral transmission. It is because the pandemic is so new and because we lack adequate knowledge of how the virus is transmitted and all of its presentations that this much needed service has become a highly difficult one to obtain [29].
This research primarily stems from those in care home settings, and not on those who are being cared for at home due to pandemic limitations. Indeed, beyond social media discussions, the majority of the difficulties that those who are cared for at home are experiencing remain unknown at this time, both from the perspective of the carer and from the perspective of the person with dementia. While some of the findings from the studies conducted in care homes will be generalizable to at home care settings, there is much that remains unknown, and a great deal that remains to be investigated. Quite simply, without conducting studies that focus expressly on in home care, it will not be possible to know the full depth of the impact of COVID-19 within this population.

\section{Pandemic Effects on Carers}

It is not only patients with dementia who are affected by the COVID19 pandemic. Some researchers predict that caregivers "pay the highest price during this crisis," more so than even neurologists, medical staff, or the dementia patients themselves. Patients with dementia are experiencing difficulties associated with receipt of care although medical professionals are making efforts to maintain care delivery to the degree possible. All individuals within our global society are faced with additional stressors, increased anxiety, and the present threat of potential exposure to the virus and illness, including both patients and their carers [30].

Caregivers, however, are experiencing even more stressors than normal. Not only do caregivers have higher stress levels during nonpandemic times, as compared to during the pandemic, they are cut off from their previous social support structures, and may even be unable to gain access to an individual to take over the provision of care due to a need to decrease the potential risk to the person with dementia. In addition, carers are being asked to integrate technologies that they themselves may not be wholly familiar with, teach their charges how to use that technology, and address all of the adverse side effects that come with the disruption of a daily routine, the integration of new activities, and the additional associated adverse behaviors that may occur as a result of these variations [28].

Other factors that must be taken into account are those who are unable to leave their homes, such as due to stay at home orders, lack of respite from the provision of care, and a lack of coping structures to facilitate carers of people with dementia who are angry, violent, disinhibited, or who have become so as a result of the pandemic. There are no care structures in place to provide supports for these concerns. Likewise, there is a need to explore the differences in provision of care based on home structure, such as whether the carer is solely providing care for a person with dementia, whether there are others in the home, and whether the carer has children to take care of as well. Still further, there is a need to identify who is serving as the carer: is it the partner, husband, or wife of the person with dementia, their child or children, or someone else?

Meanwhile, if those additional stressors were not enough, carers are also experiencing the additional pandemic stressors associated with rising costs of living, rising food prices, the potential for loss of income from jobs as more and more companies furlough their workers, loss of pandemic assistance, and increased fear of exposure as they access the now limited healthcare system that they used to rely on to facilitate the provision of care for the patient with dementia they are responsible for providing care to, all the while attempting to navigate ways to address that increased risk of exposure. This may extend to difficulties gaining access to treatment for new comorbidities that may arise unrelated to COVID-19. Caregivers of people with dementia have higher stress levels than those without; the pandemic has introduced additional stressors regarding health status, combined with concerns over failing global economies. Without access to supportive services, caregivers are receiving the brunt of all of these stressors, without any way to mitigate those stressors, placing them in the worst position of all. 
Simply put, there are neither the procedures nor the resources necessary to facilitate the transition to operating as a caregiver during pandemic times $[28,30]$.

\section{Summary}

Age is one of the greatest risk factors for both dementia and COVID19. Still further, public health guidelines may be difficult to follow for those diagnosed with some form of cognitive impairment, as for many, they may be unable to remember or to maintain basic practices designed to mitigate risk and reduce the spread of the virus. This places carers in a particularly difficult position, given that they are the ones who must take responsibility for ensuring that people living with dementia are adhering to all required procedures put in place to attempt to reduce the pandemic's spread. The problem of addressing dementia care during the COVID-19 pandemic is a global problem, yet like the pandemic there is no clear solution. There is no one size fits all approach that can be applied to the situations carers face due to individual differences in the care experience, individual differences in presentation of behavior in the person with dementia, and variations in coping skills in the carer and the patients themselves, among other considerations.

Furthermore, unlike the pandemic, there are no clear best practices that have been designed to address the provision of care within this population during the pandemic due to focus on other aspects of consideration during the pandemic. Because there is a need to address large-scale issues associated with pandemic management before focusing on individual groups within society, research simply has not yet reached the point of addressing small group needs within the population. Yet, in spite of the need to prioritize attention to the bigger picture before focusing on its individual components, there is still a need to explore these concerns and considerations. It is this researcher's hope that this review will increase awareness of these unknown factors and thereby promote increased attention to these concerns.

Lack of uniform practices regarding delivery of care, alternatives for lost services, and even the use of telehealth technologies are essential if this population is to continue to receive a basic level of care. If society is to ensure the continued care and protection of its most vulnerable populations, there is a marked need to identify the measures that are most effective in the provision of care to the dementia patient population during this healthcare crisis. By highlighting these gaps within the literature, it is hoped that research efforts will start to shift to these areas, resulting in improvements for individuals diagnosed with dementia and their carers.

\section{References}

1. Cucinotta D and Vanelli M. WHO declares COVID-19 a pandemic (2020) Acta Bio-medica 91: 157-160. https://doi.org/10.23750/abm.v91i1.9397

2. Grech V. Unknown unknowns-COVID-19 and potential global mortality (2020) Early Human Develop 144: 105026. https://doi.org/10.1016/j.earlhumdev.2020.105026

3. Lahiri D and Ardila A. COVID-19 pandemic: a neurological perspective (2020) Chreus 12: 7889. https://doi.org/10.7759/cures.7889

4. Markus H and Brainin M. COVID-19 and stroke-a global world stroke organization perspective (2020) Int J Stroke 15: 361-364. https://doi.org/10.1177/1747493020923472

5. Schneider E. Failing the test-The tragic data gap undermining the U.S. pandemic response (2020) New England J Med 383: 299302. https://doi.org/10.1056/NEJMp2014836

6. Brown E, Kumar S, Rajji T, Pollock B and Mulsant B. Anticipating and mitigating the impact of the COVID-19 pandemic on Alzheimer's disease and related dementias (2020) American J Geriatric Psyc 28: 712-721.

https://doi.org/10.1016/j.jagp.2020.04.010

7. Steinman M, Perry L and Perissinotto C. Meeting the care needs of older adults isolated at home during the COVID-19 pandemic (2020) JAMA Int Med 180: 819-820.

https://doi.org/10.1001/jamainternmed.2020.1661

8. U.S. Department of Health and Human Services [HHS]. (2020, March). HHS provides expanded guidance to nursing facilities to prevent spread of COVID-19 in Texas.

9. Shea $\mathrm{Y}$ and DeKosky S. Time-to-change: Dementia care in COVID-19 (2020) Psychogeriatrics 20: 792-793.

https://doi.org/10.1111/psyg.12576

10. Mayo Clinic. (2020, April 19). Causes of dementia.

11. Allen A, Buckley M, Cryan J, Chorcorain A, Dinan T, et al. Informal caregiving for dementia patients: the contribution of patient characteristics and behaviours to caregiver burden (2020) Ageing 49: 52-56. https://doi.org/10.1093/ageing/afz128

12. Feast A, Orrell M, Charlesworth G, Melunsky N, Poland F, et al Behavioural and psychological symptoms in dementia and the challenges for family carers: Systematic review (2016) British J Psychiatry 208: 429-434. https://doi.org/10.1192/bjp.bp.114.153684

13. Miller L, Whitlatch $\mathrm{C}$ and Lyons K. Shared decision-making in dementia: A review of patient and family carer involvement (2014) Dementia 15: 1141-1157. https://doi.org/10.1177/1471301214555542

14. Russell G. Dementia diagnosis and white lies: A necessary evil for carers of dementia patients? (2018) Int J Care and Caring 2: 133-137. https://doi.org/10.1332/239788217X15090949539964

15. Comas-Herrera A and Zalakain J. Mortality associated with COVID-19 outbreaks in care homes: Early international evidence (2020) International Long Term Care Policy Network.

16. Hanratty B, Burton J, Goodman C, Gordon A and Spilsbury K. Covid-19 and lack of linked datasets for care homes (2020) BMJ 369: 2463. https://doi.org/10.1136/bmj.m2463

17. Oliver D. Let's not forget care homes when COVID-19 is over (2020) BMJ 369: 1629. https://doi.org/10.1136/bmj.m1629

18. Sinclair A, Dhatariya K, Burr O, Nagi D, Higgins J, et al. Guidelines for the management of diabetes in care homes during the COVID-19 pandemic (2020) Diabetic Med 37: 1090-1093. https://doi.org/10.111/dme.14317

19. Martin-Jimenez P, Munoz-Garcia M, Seoane D, Roca-Rodriguez $\mathrm{L}$, Garcia-Reyne A, et al. Cognitive impairment is a common comorbidity in COVID 19 deceased patients. a hospital-based retrospective cohort study (2020) MedRxiv. https://doi.org/10.1101/2020.06.08.20125872

20. Office for National Statistics. (2020, July 3). Deaths involving COVID-19 in the care sector, England and Wales: Deaths occurring up to 12 June 2020 and registered up to 20 June 2020 (provisional)

21. Cipriani $G$ and Di Fiorino M. Access to care for dementia patients suffering from COVID-19 (2020) American J Geriatric Psyc, 28: 796-797. https://doi.org/10.1016/j.jagp.2020.04.009

22. Bhaskar S, Bradley S, Israeli-Korn S, Menon B, Chattu V, et al Chronic neurology in COVID-19 era: clinical considerations and recommendation from the reprogram consortium (2020) Frontiers Neurol 11: 664. https://doi.org/10.3389/fneur.2020.00664

23. Azarpazhooh M, Amiri A, Morovatdar N, Steinwender S, Ardani, A, et al. Correlations between COVID-19 and burden of dementia: An ecological study and review of literature (2020) J Neuro Sci 416: 117013.https://doi.org/10.1016/j.jns.2020.117013

24. Korczyn A. Dementia in the COVID-19 period (2020) J Alzheimer's disease 4: 1071-1072. https://doi.org/10.3233/JAD200609

25. Pennycook G, McPhetres J, Bago B and Rand D. Predictors of attitudes and misperceptions about COVID-19 in Canada, the U.K., and the U.S.A. (2020) PsyArXiv 1-26. https://doi.org/10.31234/osf.io/zhjkp 
26. Mann D, Chen J, Chunara R, Testa P and Nov O. COVID-19 transforms health care through telemedicine: evidence from the field (2020) J American Med Informatics Associ 27: 1132-1135. https://doi.org/10.1093/jamia/ocaa072

27. Padala S, Jendro A and Orr L. Facetime to reduce behavioral problems in a nursing home resident with Alzheimer's dementia during COVID-19 (2020) Psych Res 288: 113028. https://doi.org/10.1016/i.psychres.2020.113028

28. Wang H, Li T, Barbarino $\mathrm{P}$, Gauthier S, Brodaty $\mathrm{H}$, et al. Dementia care during COVID-19 (2020) Lancet 395: 1190-1191. https://doi.org/10.1016/S0140-6736(20)30755-8
29. Marchini L and Ettinger R. COVID-19 and geriatric dentistry: What will be the new-normal? (2020) Brazilian Dental Sci 23: 18. https://doi.org/10.14295/bsd.2020.v23i2.2226

30. Migliaccio $\mathrm{R}$ and Bouzigues A. Dementia and COVID-19 lockdown: More than a double blow for patients and caregivers (2020) J Alzheimer's Disease Rep 4: 231-235. https://doi.org/10.3233/ADR-200193 